\title{
Elaboración del guion instruccional mediante la herramienta didáctica del recurso educativo digital ${ }^{1}$
}

\author{
Elaboration of the Instructional Guideline Through the Didactic Tool \\ of Digital Educational Resources
}

Aida Gómez Suárez ${ }^{2}$

Fecha de recepción: 4 de enero de 2017 Fecha de aprobación: 4 de marzo de 2017

\section{RESUMEN}

Uno de los más destacables enfoques en el uso de las tecnologías de la información y la comunicación (TIC), en el proceso de la enseñanza-aprendizaje, es poder implementar recursos educativos digitales (RED) que favorezcan la autonomía educativa en el estudiante y que le permitan al profesor centrarse en áreas de conocimiento más significativas y específicas.

En este artículo se exponen los resultados de un proyecto de investigación que tuvo por finalidad evaluar el RED como herramienta didáctica en la elaboración del guion instruccional que permite el diseño de recursos educativos digitales, como apoyo a la clase presencial. Esta investigación se contextualizó en la labor en clase presencial de docentes universitarios de la Universidad Jorge Tadeo Lozano (Bogotá, Colombia). Los resultados permitieron determinar la percepción de los docentes sobre el RED como herramienta didáctica y sustentar estrategias para su elaboración y uso.

1 Artículo resultado de la investigación Recurso educativo digital como herramienta didáctica para la elaboración del guion instruccional, aprobada por la Universidad de la Sabana. Bogotá, Colombia. DOI: http://dx.doi. org/10.15332/s1909-0528.2017.0002.06

2 Docente-investigadora de la Facultad de Artes y Diseño de la Universidad Jorge Tadeo Lozano. Bogotá, Colombia. Magíster en Informática Educativa de la Universidad de la Sabana. Bogotá, Colombia. Correo electrónico: aida. gomez@utadeo.edu.co 
Palabras clave: tecnología, formación docente, recursos educativos digitales, guion, diseño, multimedia, hipertexto, herramienta.

\section{Abstract}

One of the most important approaches in the use of information and communication technologies (ICTs) in the teaching-learning process is to be able to implement digital educational resources (REDs) that favor educational autonomy in the student and that Allow the teacher to focus on more meaningful and specific areas of knowledge.

This article presents the results of a research project whose purpose was to evaluate the RED as a didactic tool in the elaboration of the instructional guide that allows the design of digital educational resources, as a support to the classroom. This research was contextualized in the classroom work of university professors of the University Jorge Tadeo Lozano (Bogotá, Colombia). The results allowed to determine the perception of the teachers on the RED as a didactic tool and to sustain strategies for their elaboration and use.

Keywords: Technology, Teacher training, Digital educational resources, Script, Design, Multimedia, Hypertext tool. 


\section{INTRODUCCIÓN}

De conformidad con Flórez (2012, p.44), "no hay duda de que la conectividad y el acceso a nuevas tecnologías de información han crecido vertiginosamente en Colombia durante los últimos años y que aunque aún nos falta bastante para estar al nivel de países líderes como los europeos, es indudable que vamos por un buen camino".

Atendiendo lo manifestado por Sarmiento (2016, p. 99), en el sentido de considerar "la necesidad inminente de re-dimensionar el conocimiento y su reconocimiento como factor determinante en las instituciones de educación superior, la gestión del conocimiento se presenta como aquella alternativa interesante que ejecuta tan difícil tarea". Desde la citada perspectiva, el avance de las tecnologías de la información y las comunicaciones, comúnmente denominadas TIC, ha generado muchas discusiones en torno a su uso e implementación en los distintos ámbitos de la sociedad. En el campo de la educación, también se asumen posiciones sobre las ventajas y desventajas, basadas en reflexiones y evaluaciones acerca de hasta qué punto son efectivas en los procesos de aprendizaje, y sobre quiénes son los actores responsables de su proyección, implementación y consolidación.

Las instituciones educativas han emprendido acciones para adaptarse a los cambios necesarios a fin de mejorar sus procesos educativos en una sociedad cada vez más tecnificada y, con ello, posicionarse como instituciones de alta calidad. Una de las acciones más destacadas ha sido la ampliación de la infraestructura tecnológica, orientada a ofrecer aplicaciones sofisticadas y modernas a la comunidad educativa, cuyos recursos tecnológicos y uso de estas herramientas se deben apoyar en la formulación de planes de capacitación para los profesores, y no solo estos, también para los directivos académicos y administrativos que son parte esencial en la proyección e implementación de estas tecnologías, como parte integral de la gestión educativa que realizan.

El desarrollo de la competencia ética y técnica en el directivo de instituciones educativas, debe reflejarse en la gestión educativa, en el modo como promueve el comportamiento virtuoso de la comunidad educativa para 
asumir los cambios y en el aprovechamiento responsable de las TIC para el mejoramiento de la calidad educativa y el perfeccionamiento del ser humano (Sandoval, 2010).

Por lo anterior, la inclusión de tecnología en las instituciones educativas implica una reflexión en torno a la pertinencia del currículo y a la reconfiguración de los roles dentro del proceso de enseñanza-aprendizaje, con el fin de ajustarlo a las necesidades emergentes; al respecto Campuzano (1992), considera: "Es muy importante para la institución escolar de cada país, que las nuevas tecnologías se integren adecuadamente, y dentro de sus posibilidades, en sus dinámicas educativas, administrativas, culturales, etc. Son herramientas poderosas que deben ponerse al alcance de todos los individuos".

Sin embargo, hay que tener en cuenta que hablar de tecnología no evoca, necesariamente, el concepto de innovación. La Real Academia Española (RAE) define tecnología como "el conjunto de teorías y técnicas que permiten el aprovechamiento práctico del conocimiento científico"; un aprovechamiento que ha sido constante en el progreso de la humanidad y que se hace de forma cotidiana, sin que estas experiencias puedan ser consideradas "nuevas". La relación entre el uso de la tecnología y la percepción de lo "nuevo" se debe, según Lara (2011, p.7), a que "la tecnología hace pensar que al trabajarla se está produciendo 'lo nuevo' y esto se traspasa directamente a nuestra mente para sentir ahora que lo tecnológico es lo nuevo y a la vez que lo nuevo es un mayor valor de las cosas"; pero lo verdaderamente importante es la reflexión por los procesos que conllevan a resultados, sobre todo en el campo de la educación, en el que "el auténtico reto que nos espera no es, pues, la tecnología, sino para qué la utilizamos" (Drucker, 1993, p. 193).

En relación con esto, el reto de integrar las TIC en los procesos de enseñanzaaprendizaje en las instituciones de educación superior ha generado tantas expectativas como obstáculos. Uno de esos obstáculos es precisamente su uso, y esto implica, según Cabero, que "los contextos educativos actuales deberán apostar por una integración crítica, en la cual se defina el qué, por qué y para qué de su implementación y aprovechamiento" (2007, p. 5). 
Es importante anotar que entendemos por TIC "las tecnologías, la información y las comunicaciones conectadas por Internet, lo cual tiene un efecto integrado significativo en la globalización y la sociedad de la información, lo cual se manifiesta como un fenómeno a través del cual se produce el conocimiento de la humanidad a nivel mundial de los eventos económicos, culturales, políticos, etc." (Moreno \& Velásquez, 2015, p. 139).

Para lograr una verdadera integración crítica de la tecnología en los contextos educativos es necesario centrar la atención en uno de sus actores principales, el docente, y preguntarse cuál es rol que este debe asumir frente al uso de las TIC en los escenarios tradicionales, toda vez que, según Duart \& Sangrá (2000), la formación en entornos virtuales requieren de una organización diferente a la de la formación presencial; por lo que es necesario revisar la tendencia en las instituciones de educación superior a reproducir en los nuevos escenarios formativos prácticas convencionales de la clase presencial con las mismas estrategias, pero con el uso de algunas herramientas tecnológicas, "el profesor continúa siendo el único referente válido de transmisión del conocimiento, y centrándose básicamente en las nuevas tecnologías para ampliar el alcance de sus clases” (Duart \& Sangrá, 2000, p. 9).

Para afrontar esta situación, que afecta el uso crítico didáctico y pedagógico de las tecnologías, algunos expertos (Cabero, 2007), consideran que se debe volver a configurar el escenario educativo, y esto requiere de formación y perfeccionamiento de la comunidad, tanto académica como administrativa; también se hace necesario reformar funciones, estructuras organizativas y metodologías, para que las tecnologías sean un medio más en el proceso formativo, no el fin último.

Las principales exigencias en este proceso es que las instituciones educativas deben resignificar el rol del docente, comprender que no es el dueño absoluto del conocimiento y que debe convertirse en el mediador de la construcción del conocimiento como encargado de llevar al estudiante a "la zona de desarrollo próximo", definida como: "La distancia entre el nivel real de desarrollo, determinado por la capacidad de resolver independientemente un problema, y el nivel de desarrollo potencial, determinado a través de la resolución de un problema bajo la guía de un adulto o en colaboración con otro compañero más capaz” (Vygotsky, 1988, p. 133). 
Sin embargo, existe una serie de factores que obstaculizan dicha transformación; en el estudio de caso que se realizó en la investigación en la que se sustenta este artículo, profesores de la Universidad de Bogotá Jorge Tadeo Lozano, se puede evidenciar cierta resistencia en cuanto a la implementación de las TIC como apoyo a la clase presencial; esto surge, seguramente, del desconocimiento que existe en torno a la implementación de las mismas, teniendo en cuenta que no se trata solo de implementarlas, sino que también es necesario saber qué se va a hacer, para quién se va hacer y cómo se va a hacer.

Dentro de las características del perfil del docente tadeísta, contempladas en el plan de capacitación profesoral, uno de los puntos se relaciona con el dominio TIC en el proceso de enseńanza-aprendizaje; además el programa de desarrollo incluye la formación en TIC. El docente es responsable de construir su propio material didáctico; sin embargo, la mayoría de ellos no ha tenido una formación gráficovisual y mucho menos una formación multimedial, y de hecho su vinculación no implica que deban tenerla. Las instrucciones que reciben los docentes, con respecto al aula virtual, orientan que los materiales didácticos se deben elaborar, guiados por el syllabus de la asignatura a cargo de ellos, y que el fin del aula virtual es apoyar la clase presencial.

Uno de los propósitos del syllabus definido, por la Vicerrectoría Académica, consiste en apoyar el trabajo presencial y no presencial del estudiante mediante la incorporación del Ambiente Virtual de Aprendizaje Tadeísta (AVATA). El docente elabora el programa a la luz del syllabus de la asignatura, ejerciendo en pleno su responsabilidad, creatividad y capacidad de innovación educativa. En relación con este ejercicio, la investigación permitió determinar que la tendencia que identifica a las aulas virtuales de la Universidad Jorge Tadeo Lozano es que son repositorios de documentos portables (comúnmente conocidos en el ámbito educativo como objetos de información), y que no se aprovechan plenamente los recursos de dichas aulas para desarrollar actividades o ejercicios de realimentación. Esto conlleva al abandono del aula virtual por parte del estudiante, y el docente continúa con su metodología habitual; la reprografía (fotocopia) sigue vigente como "recurso educativo" en las prácticas académicas. 
Por lo anterior, la investigación realizada se orientó al diseño, desarrollo e implementación de un RED que le brinde al profesor conocimientos adecuados con relación al guion instruccional, como integrador entre el contenido temático (unidad temática) de una asignatura y su dinamización, en un recurso educativo digital, con el fin de contribuir al uso de las TIC como apoyo a la clase presencial. Adicionalmente al concepto relacionados al guion instruccional, los RED, también se abordaron los conceptos asociados a los contenidos digitales, así como al equipo de trabajo que interviene en el proceso de producción; en este aspecto se hizo mucho énfasis en el rol del docente como experto temático.

En dicho trabajo de investigación participaron docentes de diferentes áreas del conocimiento, lo que permitió identificar variadas dinámicas de trabajo, así como la facilidad o dificultad para la comprensión de los conceptos asociados al guion instruccional y al RED. De igual forma, el RED contribuyó al aprendizaje autónomo de los docentes, teniendo en cuenta que fueron ellos los autores responsables de su proceso formativo, lo cual se hizo evidente en la entrega del guion instruccional ya elaborado.

\subsection{LAS TECNOLOGÍAS DE LA INFORMACIÓN Y LAS COMUNICACIONES EN EL ÁMBITO EDUCATIVO}

De conformidad con Puentes (2016, p. 19), "el desarrollo y la apropiación de las TIC han marcado el rumbo de las naciones en la medida en que estas han asumido dichas tecnologías en todos los niveles. Los innumerables beneficios y promesas de la apropiación de estas tecnologías por parte de los ciudadanos, ante todo de las empresas que componen una economía, al parecer aún se hacen esperar”.

Las tecnologías de la información y las comunicaciones (TIC) son una de las principales características de la actual sociedad del conocimiento, por lo que es inevitable que se exija un uso adecuado de estas en las instituciones educativas como requerimiento para el aseguramiento de la calidad educativa que sustenta la acreditación de estas; pero, en la mayoría de los casos, no se invierte lo suficiente en el trasfondo y la implicaciones de estos usos, lo que no ha impedido, sin embargo, 
el interés de los docentes por alcanzar competencias informáticas que les permitan llevar a cabo su actividad docente, con el fin de ajustarse a las exigencias educativas actuales.

Este interés por las tecnologías de la información y la comunicación TIC en el ámbito educativo se asocia a los cambios que se han venido presentando en las prácticas de la enseńanza y el aprendizaje. Para las instituciones educativas, la implementación de estos recursos les permite sustentar el mejoramiento de sus proyectos educativos institucionales (PEI) y acoger las actuales propuestas formativas, de tal manera, que les permita ser competitivas en la actual sociedad del conocimiento; una sociedad en la que Internet se constituye en un apoyo didáctico para la enseńanza, puesto que acerca al estudiante a contextos formativos que le permite desarrollar una serie de procesos psicológicos dentro de la cual se pueden encontrar la creatividad, la curiosidad, el juicio crítico y la imaginación (Ortiz \& Aguilar, 2010).

Esto hace muy importante reflexionar sobre el uso apropiado de las TIC como medio didáctico a través del cual se construye conocimiento, pero no necesariamente donde se concibe, para no caer en el error de suponer que estas pueden resolver las diferentes problemáticas que se presentan en el ambiente educativo.

Los espacios educativos también se encuentran en constante transformación, las nuevas estancias educativas se han reflejado en centros virtuales de aprendizaje. Sin embargo, estos nuevos escenarios requieren de una reflexión hacia el uso e incorporación de las tecnologías, los contextos educativos actuales deberán apostar por una integración crítica, en la cual se defina el qué, el por qué y el para qué de su incorporación y aprovechamiento (Cabero, 2007, p. 2).

En la sociedad del conocimiento, la educación ha pasado a ocupar un lugar central, pues "el conocimiento es la mercancía más valiosa, y la educación y la formación son las vías para adquirirlo” (Coll, 2004, p. 3).

La centralidad de la educación en la sociedad de la información, junto con las nuevas posibilidades de acceso a la información y el conocimiento que ofrecen las TIC, ha llevado a profesores, investigadores, gestores, planificadores, responsables políticos y 
profesionales de la educación en general, a señalar cada vez con mayor insistencia, la necesidad de una revisión en profundidad del papel, las funciones y la organización de los sistemas y servicios educativos (Coll, 2004, p. 3).

Dicha centralidad de la educación y la formación "ha estado acompañada de un protagonismo igualmente creciente de las TIC en los procesos educativos y formativos" (Coll 2004, p. 3). Las TIC transforman los ambientes educativos convencionales, pues gestan nuevas prácticas educativas en el proceso de enseńanzaaprendizaje; sin embargo, y como cita Cabero "hay que ver las tecnologías como medio y recurso didáctico, más no como la panacea que resolverá las problemáticas dentro del ámbito educativo" (Cabero, 2007, p. 2). Dicha incorporación de las TIC en el ámbito educativo conlleva a formular preguntas como: ¿están preparados los actores de los procesos educativos para afrontar los cambios que implican?, ¿las instituciones educativas son conscientes de los procesos que se deben afrontar para incorporarlas en la práctica docente? y ¿los docentes están en capacidad de asumir los cambios con conocimiento y responsabilidad?

Es claro que el avance de la sociedad del conocimiento ha desencadenado un acelerado desarrollo de herramientas informáticas que le permiten al ser humano acceder a la información y los saberes de muchas formas; pero, las herramientas informáticas son solamente eso: herramientas, y en esa medida son una pequeña parte de la transformación de la práctica educativa; pese a ello, en muchos casos son abordadas como la solución al quehacer educativo, desconociendo la realidad de los contextos en su implementación. Para afrontar estos retos, es necesario que los docentes se apropien de las herramientas, a partir de capacitaciones que sustenten sus competencias en el uso de estas en ajuste con los lineamientos de los modelos pedagógicos institucionales, en una sociedad del conocimiento que ha derivado en procesos formativos autónomos, rica en contenidos y que ha facilitado el acceso a la información en cualquier momento y desde cualquier lugar. En tal escenario educativo se revela la importancia del diseño digital que "es la concepción de proyectos de comunicación que utilicen eficazmente todo el potencial de los medios interactivos" (Orihuela \& Santos, 2000, p. 37). 


\subsection{El ROL DEL PROFESOR Y EL PAPEL DE LAS INSTITUCIONES EDUCATIVAS EN LAS TRANSFORMACIONES DE LOS ESCENARIOS FORMATIVOS}

La educación se concibe desde una amplia gama de perspectivas. Algunos la perciben solo como un proceso de enseńanza-aprendizaje; otros, la visualizan como una acción laboral; otros, como un servicio y otros, como un derecho. La enseñanza y el aprendizaje son uno solo; pues no puede hablarse de enseñar sin que alguien aprenda; aunque, sí es posible hablar de lo contrario, es decir, de alguien que aprende, sin que nadie le enseñe. Estos dos procesos (la enseñanza y el aprendizaje) hacen parte del desarrollo humano, de la formación de cada una de las personas; pero cuando se habla de enseñanza-aprendizaje, se está hablando de un proceso establecido dentro de un plan institucional con un propósito formal y estrictamente definido.

Utilizar técnicas, modelos o herramientas educativas que no son acordes con la cultura institucional o con las características de los educandos y de los profesores que comparten dicho proceso es aplicar actividades que son o resultan insulsas a la hora de verificar si se está enseñando y si se está aprendiendo. La didáctica requiere de mucho más que generar espacios para que se reúnan las personas a construir conocimiento acerca de un tema específico; requiere una planeación estratégica de los contextos académicos y, en las actuales condiciones, es esta la base para aprovechar las verdaderas ventajas de las herramientas que ofrecen las TIC, sobre las que se dice que es mucho más "sencillo" el aprendizaje si se utilizan, que si no; afirmación que exige comprender lo que realmente sucede y se requiere al implementarlas.

Utilizar las TIC, en el proceso enseñanza-aprendizaje, puede facilitar que el aprendizaje sea verdaderamente significativo o autónomo; pero puede poner en aprietos a uno de sus actores relevantes: los profesores; pues el uso de estas tecnologías, indiscutiblemente, exige una preparación y capacitación para los docentes, que no solo consiste en alcanzar la competencia en el manejo de herramientas o recursos; va mucho más allá, también consiste en la forma en la que el docente debe afrontar una nueva postura frente a estas. En este sentido, el profesor debe estar en la capacidad de replantear y transformar su mentalidad y sus habituales procesos de enseñanza 
y las estrategias que de esta deriven, ya que debe ser quien facilite los procesos de enseńanza y quien incentive a los estudiantes a un verdadero aprendizaje.

Los docentes "deben ser capaces de usar las TIC para la enseńanza, lo cual implica diseñar, y producir cursos y materiales que puedan ser dictados mediante dichas tecnologías, en modalidades presenciales, mixtas y a distancia” (Dorrego, 2004, p. 127). Sin embargo, uno de los errores más frecuentes en el que recaen las instituciones educativas consiste en exigir al docente el replanteamiento de sus prácticas educativas sin haber elaborado un plan de capacitación docente y un plan de implementación oportuna, esto conlleva a que el docente se resista a apoyar su práctica académica con el uso de las TIC. En otros casos: "La mayor parte de los profesores se resiste al uso de tecnologías por dos razones:1) se ven como los “expertos” de sus disciplinas y creen que el uso de otros recursos quizás disminuiría sus posiciones profesionales; 2) aprendieron con lecturas y libros solamente, y no tienen modelos de cómo enseñar con tecnologías (Peter, 2004, p. 73).

Pero, ¿cuál es el rol de profesor? Pues no se trata solamente de "utilizar" técnicas o didácticas, se trata de aprovecharlas en su real magnitud, y para ello, es necesario conocerlas a profundidad, y poner a disposición todos los requisitos que son fundamentales para que el proceso de enseñanza-aprendizaje termine por aparecer como consecuencia obvia y última en el quehacer educativo. En cuanto a su rol, el docente debe replantear sus habilidades y sus competencias de tal manera que le permitan desarrollar eficazmente su práctica académica.

Sin embargo, aunque el docente es un actor principal, las instituciones, los docentes y, por supuesto, los estudiantes requieren estar integrados en la aplicación y utilización de las nuevas tecnologías de la información y de la comunicación. Si no lo están, se estará concibiendo la educación como un proceso aislado, el cual no requiere sino de plataformas digitales para que una persona se forme en un ámbito particular.

Teniendo en cuenta lo expuesto, se identifica que, a la hora de implementar el uso de las TIC a los procesos de enseńanza-aprendizaje, prima la formación del profesor. Las instituciones deben estructurar planes de capacitación profesoral acordes al modelo pedagógico de las institución y acorde con los perfiles y necesidades de cada 
profesor, y para no lanzar al docente agresivamente a la implementación de estos procesos, se deben diseñar estrategias que permitan una fácil transición a estos nuevos procesos educativos, evitando traumatismos o abandono por parte del profesor a la capacitación o al uso de las mismas. En su artículo "Las necesidades de las TIC en el ámbito educativo: oportunidades, riesgos y necesidades”, Cabero afirma:

Para nosotros, las TIC, independientemente de su potencial instrumental y estético, son solamente medios y recursos didácticos, que deben ser movilizados por el profesor cuando les puedan resolver un problema comunicativo o le ayuden a crear un entorno diferente y propicio para el aprendizaje. No son por tanto la panacea que van a resolver los problemas educativos, es más, algunas veces incluso los aumentan, cuando como por ejemplo el profesorado abandona su práctica educativa a las TIC (Cabero, 2007, p. 6).

Y concluye al respecto: "las TIC si de verdad quieren ser integradas en las acciones educativas deben hacerse invisibles y claras" (Cabero 2007, p. 6).

\section{Metodología}

Para el diseño de la investigación, se utilizó un diseño de comparación entre grupos con prueba y posprueba y dos grupos control (Hernández-Sampieri, FernándezCollado, \& Baptista-Lucio, 2006). El diseño fue una variación de un diseño de comparación entre grupos y un grupo control. No se planteó como un estudio de tipo experimental. Su medición fue de corte transaccional o transversal (aplicación o exposición al RED, o entrenamiento en la elaboración del guion instruccional). El diseño no tuvo alcances experimentales, debido a que no se pudo establecer una relación causal entre ser expuesto al RED y el posterior desempeño del guion; sin embargo, se pudo anticipar que estar expuesto al RED facilitaría la elaboración del guion instruccional y, por ende, en mediciones indirectas, el adecuado desempeño de AVATA.

El diseño tuvo una organización que diferenciaba las condiciones iniciales y las finales de los participantes —una vez expuestos al entrenamiento de elaboración 
del guion instruccional—, diferenciándolos de los no expuestos en condiciones de aprendizaje previo.

Finalmente, propendió por la validez interna del estudio en cuanto a que mantuvo equivalentes los grupos (dada su homogeneidad, número y condición previa al entrenamiento). Adicionalmente, se definió correctamente el momento de aplicación del entrenamiento o exposición al RED en cualquiera de los grupos, facilitando y controlando las variables extrańas relacionadas con la experiencia. Los grupos fueron equivalentes inicialmente, atendiendo a las recomendaciones metodológicas propias de un diseño de comparación entre grupos.

Estos grupos no fueron de asignación al azar, dada la condición de muestra por conveniencia. La conformación de los grupos correspondió a la calificación obtenida en el último periodo académico por parte de la Coordinación AVATA de la Universidad Jorge Tadeo Lozano. Esta calificación arrojó la ubicación del docente en relación con las competencias tecnológicas, a partir de su desempeño dentro del sistema AVATA. La escala de desempeño clasifica al docente en "inicial", "básico", "intermedio" y "avanzado". Los grupos seleccionados fueron conformados por docentes que calificaron en el nivel "básico" de competencias tecnológicas.

Este diseño permitió que se hiciera una correlación entre el registro inicial del AVATA y su desempeño posterior, una vez utilizado o no el recurso educativo digital, evidenciando, de esta manera, si estar bajo entrenamiento o capacitación en la elaboración de un guion instruccional, mejoraba el desempeño en competencias tecnológicas. Se hicieron varias mediciones postest, unas directas y otras indirectas. La directa fue la elaboración misma del guion instruccional y las indirectas correspondieron a la calificación por desempeño y su reporte a través de la encuesta de recolección de datos de facilitación del RED (ver tabla 1). 
Tabla 1. Diseño de comparación entre grupos con prueba y posprueba y dos grupos de control
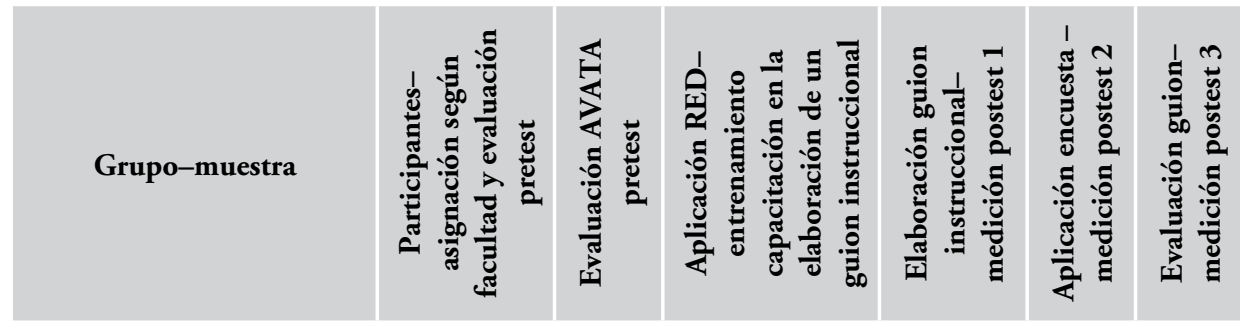

G1

Grupo de entrenamiento en guion con experiencia en el uso de herramientas Sí Sí Sí Sí Sí tecnológicas.

\section{G2}

Grupo de entrenamiento en guion sin experiencia en el uso de herramientas S Sí Sí Sí Sí Sí tecnológicas.

\section{G3}

Grupo de control en guion con experiencia en el uso de herramientas Sí Sí No Sí Sí tecnológicas

\section{G4}

Grupo de control en guion sin experiencia en el uso de herramientas tecnológicas

Sí

Nota: se muestran los momentos 1, 2 y 3 de la investigación, a través del diseño de comparación entre grupos con prueba y posprueba y dos grupos control, para todos los participantes. Se observa que todas las condiciones son iguales, excepto el entrenamiento en la elaboración del guion instruccional. Momento 1: evaluación pretest y asignación a grupos por facultad. Momento 2: entrenamiento en la elaboración del guion instruccional o no, según si se es parte de grupo control o no. Momento 3: mediciones directas e indirectas de la competencia tecnológica.

Fuente: elaboración propia. 
Los datos recolectados correspondieron a tres categorías: la primera categoría fue la calificación desde la Coordinación del AVATA de la universidad Jorge Tadeo Lozano, la cual arrojó una clasificación de carácter cualitativa ordinal (inicial, básico, intermedio y avanzado); la segunda, correspondió a la evaluación escrita de la apropiación del recurso educativo digital que recoge la información del saber qué, o mejor, de la parte conceptual y de conocimiento de una competencia; y finalmente, la tercera, correspondió a la elaboración del guion instruccional mismo, que aborda la parte operativa o de "saber cómo" de una competencia. Estas dos últimas categorías de recolección de la información también están en una clasificación de carácter cualitativo, pero nominal (logro alcanzado o logro no alcanzado).

Los datos obtenidos fueron correlacionados en cuanto a si variaron, o no, respecto del estado inicial (calificación de la Coordinación de AVATA de la Universidad Jorge Tadeo Lozano) y se revisó especialmente el cambio en procura de la mejoría respecto a este estado inicial.

\section{Resultados}

Para dar respuesta a las problemáticas relacionada con el uso de las TIC en el ámbito educativo, en la investigación cuyos resultados se exponen en este artículo, se diseñó un RED que se denominó "guion instruccional para el diseño y producción de recursos educativos digitales", como un aporte al programa de Formación en Tecnologías de la Información y la Comunicación y al Departamento de Desarrollo Profesoral de la Dirección de Innovación y Apoyo Académico de la Universidad Jorge Tadeo Lozano.

El objetivo del RED fue guiar a docentes de la Universidad Jorge Tadeo Lozano en la elaboración del guion instruccional para el diseño de recursos educativos digitales, y las metas fueron: 1) comprender las características y componentes de un RED y los procesos previos para su diseńo y producción, 2) identificar cada uno de los integrantes del equipo de trabajo, sus roles y funciones, 3) comprender el concepto de "guion instruccional" e identificar las funciones que cumple en la preproducción y producción de un RED, y 4) elaborar el guion instruccional de una asignatura a cargo. 
En el diseño del RED se contemplaron los siguientes factores: la coherencia gramatical, una información suficiente y completa, una narrativa textual e hipertextual clara, los objetivos de las actividades y actividades variadas que responden al objetivo de cada módulo.

Este recurso se dispuso en el Ambiente Virtual de Aprendizaje Tadeísta (AVATA) dentro de la categoría de capacitación docente; categoría que consigna varios cursos que corresponden a la ruta de formación docente en TIC, como capacitación en Moodle y la capacitación en herramientas digitales. Cabe resaltar que algunos de los cursos fueron planificados y diseñados por la autora de este artículo, tanto en contenidos, como en el diseño de los MED y del ambiente de aprendizaje. Cada uno de los cursos se compone de varios módulos, y el de RED está compuesto por cinco módulos (ver figura 1).

Figura1. Módulos correspondientes al curso Recursos Educativos Digitales

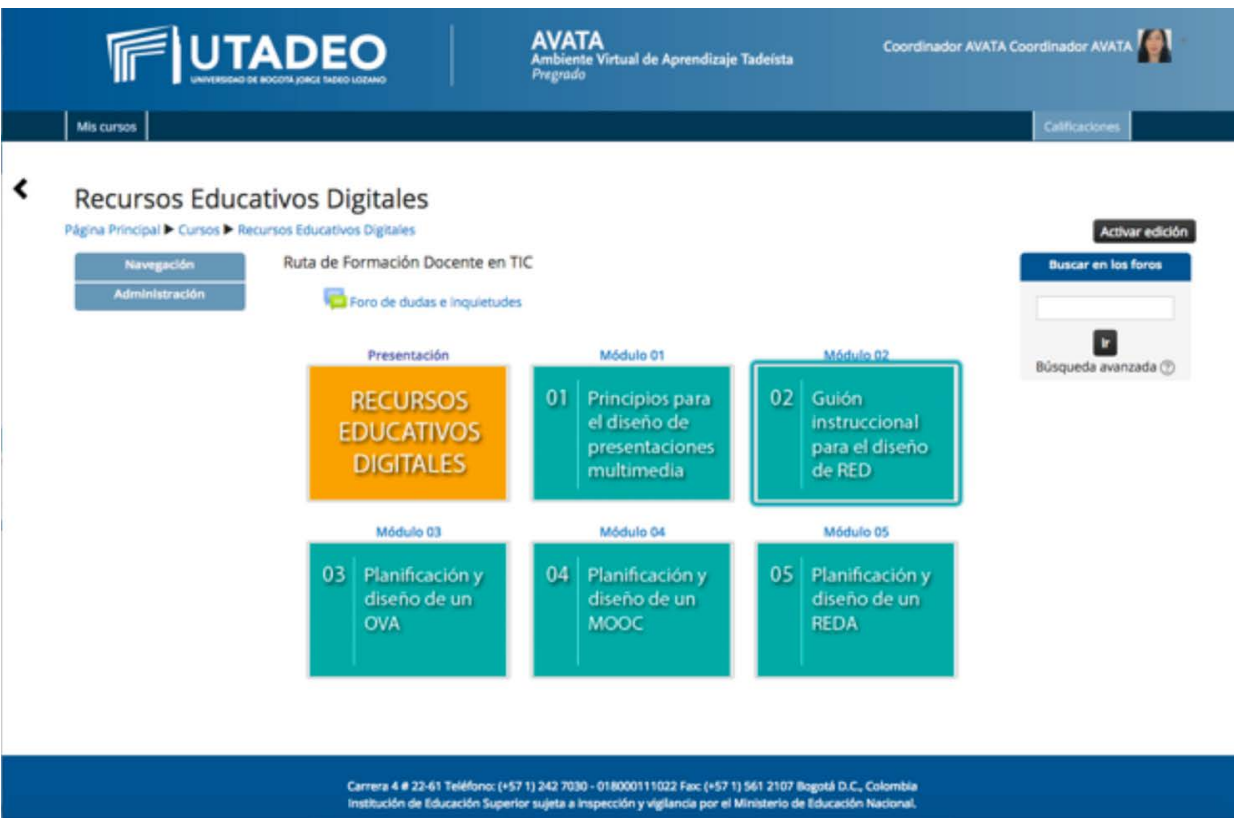

Fuente: elaboración propia con base en AVATA 
El módulo 2 corresponde al tema del guion instruccional para el diseño de recursos educativos digitales y está apoyado en el RED del guion instruccional (ver figura 2).

Figura 2. Módulo 2 correspondiente al RED del guion instruccional

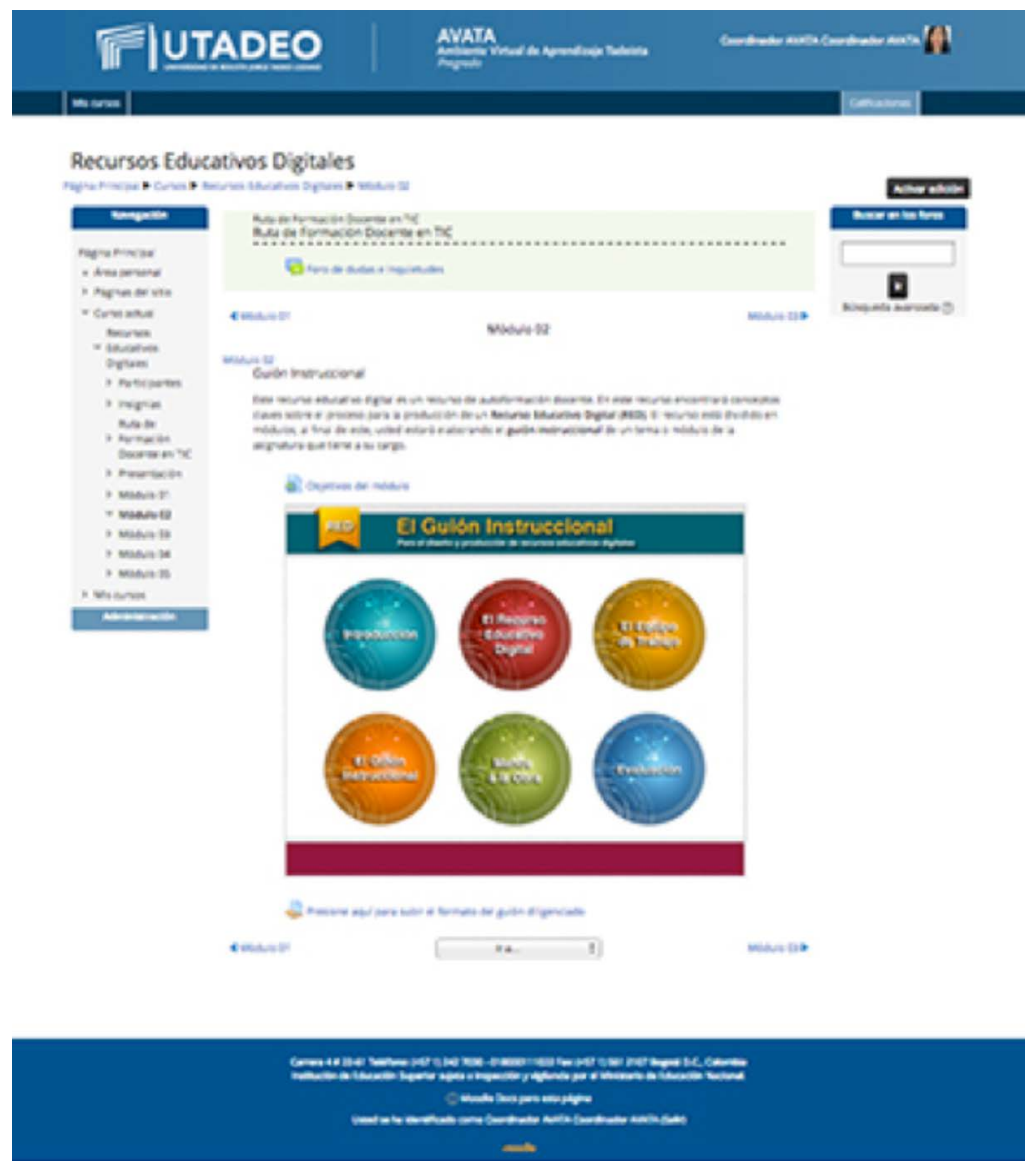

Fuente: elaboración propia con base en AVATA.

El RED está construido en una estructura hipertextual ramificada en la que se incluyen nodos subordinados para una mayor interactividad. Posee un nodo principal o de inicio (primer nivel) que da paso a seis nodos principales (segundo 
nivel) que corresponden a los seis módulos que lo componen; de cada uno de estos nodos principales (segundo nivel) se desprenden nodos de tercer y cuarto nivel (ver figura 3).

Figura 3. Estructura de organización del contenido del RED

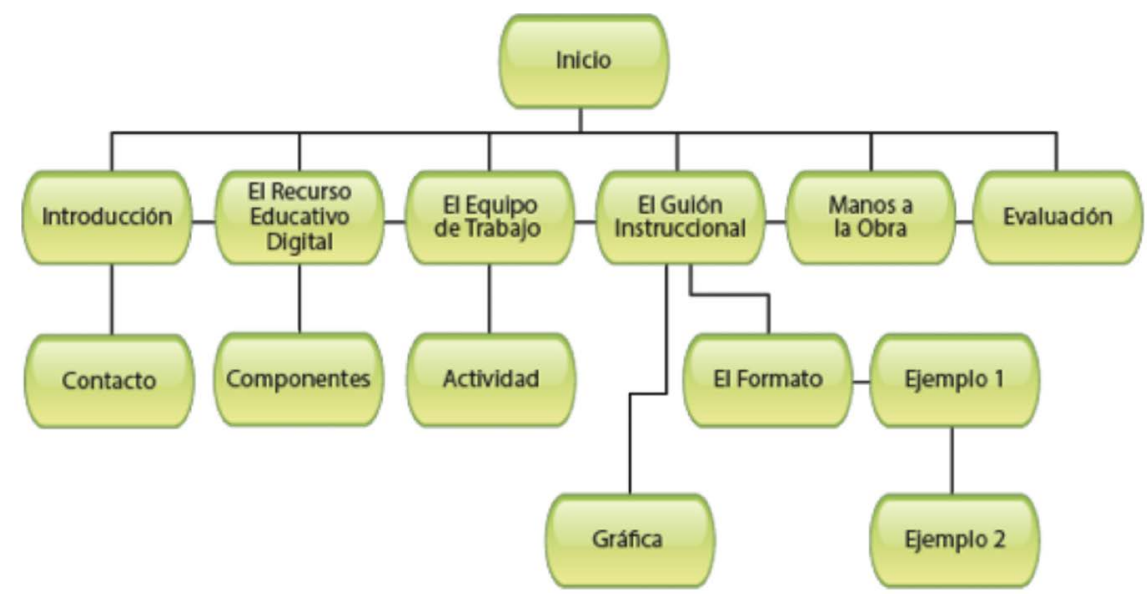

Fuente: elaboración propia.

El contenido del RED está compuesto por seis módulos y sus respectivos contenidos subordinados, dos actividades, una evaluación al final, una encuesta y un formulario de contacto.

El RED fue creado por la investigadora y autora de este artículo en su calidad de diseñadora gráfica y su desempeño actual como diseñadora multimedia, área que imparte y coordina dentro del programa de Diseńo Gráfico de la Universidad Jorge Tadeo Lozano.

En cuanto a los aspectos técnicos, este recurso está construido a través de los siguientes lenguajes de programación:

HTML: sigla que corresponde a HyperText Markup Language (lenguaje de marcado de hipertexto) a través del cual se construyen sitios web. 
CSS: sigla que corresponde a Cascading Style Sheets (hoja de estilo en cascada) este lenguaje permite definir y crear la apariencia del documento estructurado en HTML.

JQuery: está basado en Java Script, y permite adicionar animación e interacción a un documento HTML.

PHP: este lenguaje de programación permite agregar contenido dinámico a un contenido web.

Con respecto a los aspectos gráficos y de diseño, el recurso en su inicio permite la entrada a los seis módulos a través de seis botones (ver figura 4):

Figura 4. Pantalla de inicio del RED, menú y botones de navegación

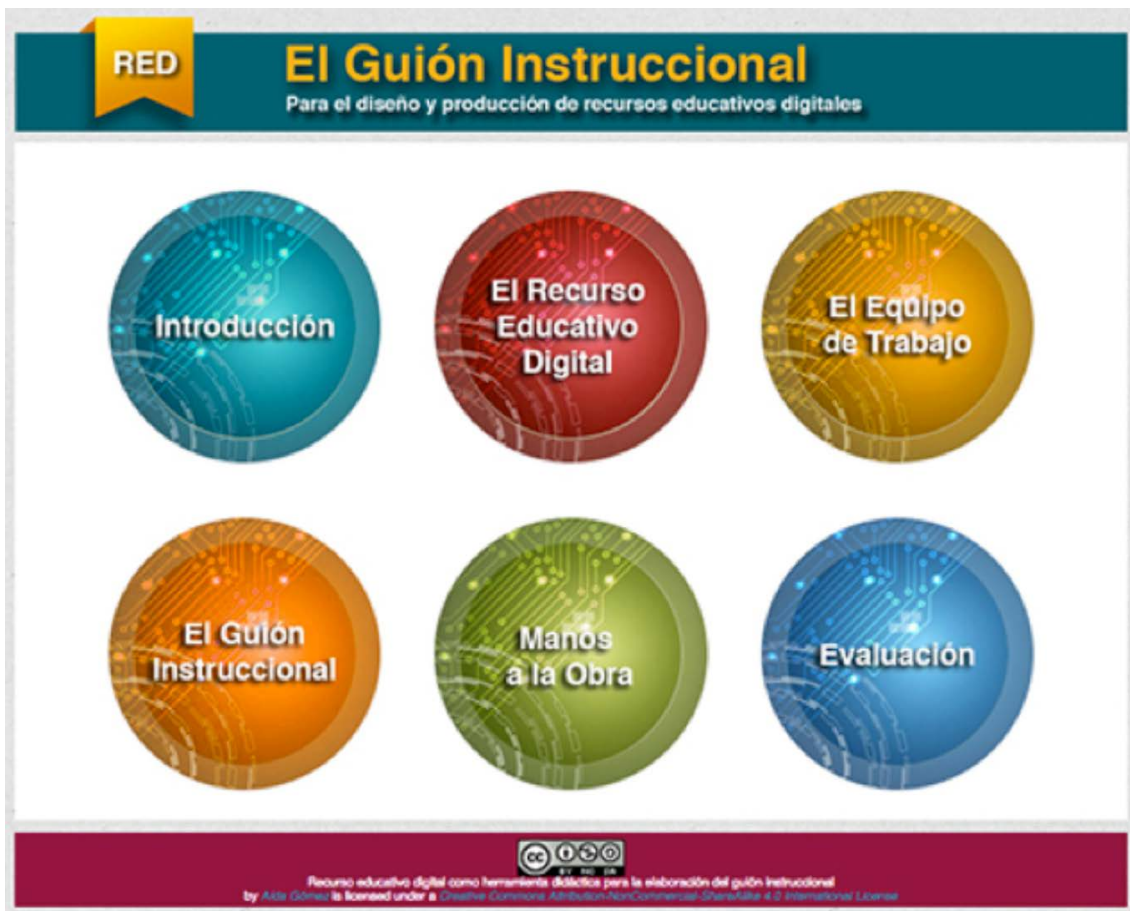

Fuente: elaboración propia 
Los módulos se organizan en los siguientes contenidos: introducción, recurso educativo digital, equipo de trabajo, guion instruccional, manos a la obra y evaluación.

El módulo1, de introducción, contiene la bienvenida y la presentación del RED.

El Módulo 2 tiene como objetivo dar a conocer, con ejemplos reales, el concepto de RED, de tal manera que al llegar al módulo 4 se entienda de manera clara y objetiva el concepto de guion instruccional. En este, se define qué es un RED (ver figura 5).

Figura 5. Pantalla de definición del guion instruccional

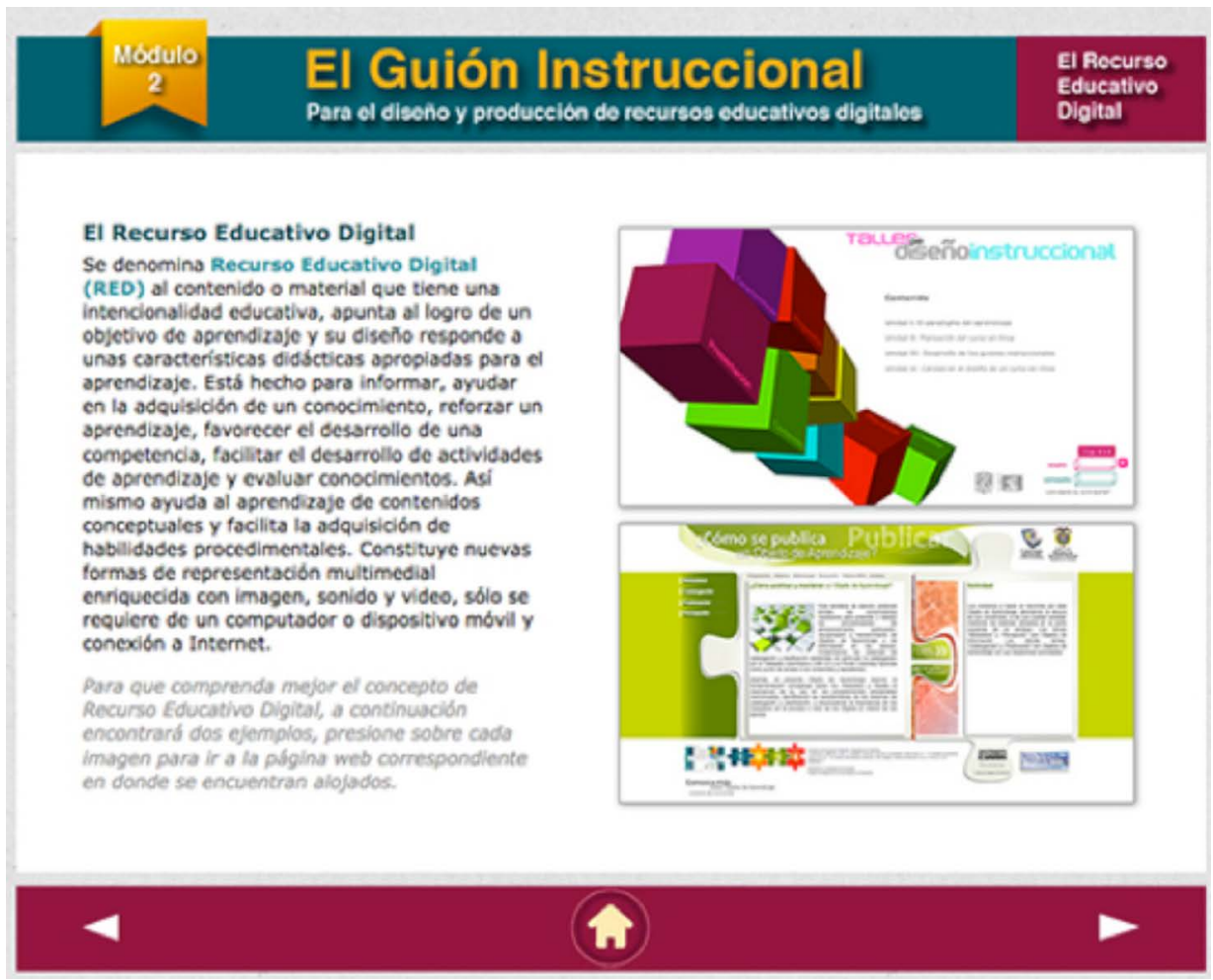

Fuente: elaboración propia 
Para apoyar la definición, se incluyen en la pantalla dos imágenes relacionadas temáticamente con el tema del guion instruccional, que representan ejemplos de recursos educativos digitales; el primero corresponde a la presentación de un curso sobre diseño instruccional para profesores de la Universidad Nacional Autónoma de México (UNAM); este recurso está disponible solo para profesores adscritos a la UNAM. El segundo corresponde a un RED sobre la gestión de objetos virtuales de aprendizaje que pertenece al Ministerio de Educación Nacional de Colombia y el portal Colombia Aprende, este recurso es abierto y se puede explorar en su totalidad. En la página 2, del módulo 2, se describen y definen los componentes de un recurso educativo digital (ver figura 6).

Figura 6. Componentes del RED

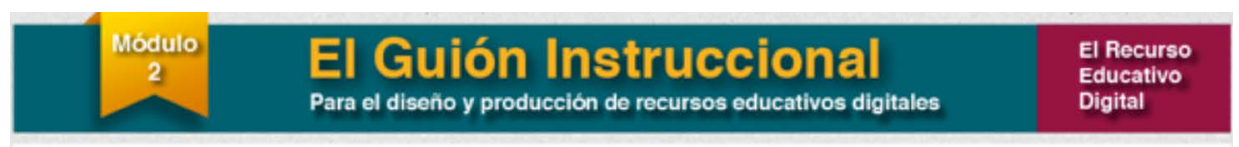

Componentes de un Recurso Educativo Digital

Después de haber explorado los recursos de ejemplo, podemos concluir que un recurso educativo digital tiene cuatro componentes: contexto, contenidos, actividades y evaluación. Ublquese sobre la imagen para ver la descripción de cada concepto.

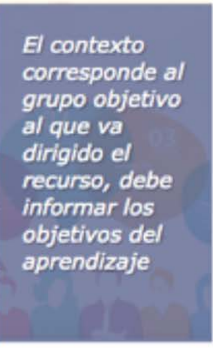

CONTEXTO

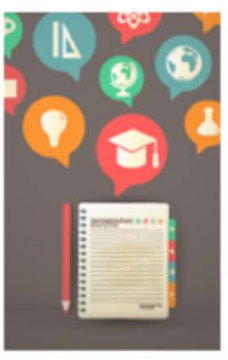

CONTENIDOS

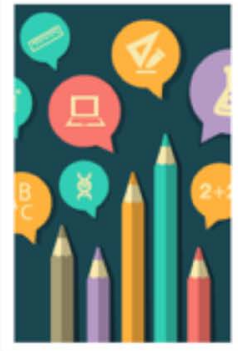

ACTIVIDADES

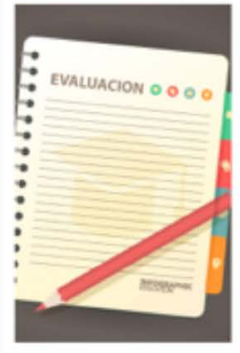

EVALUACTÓN

\section{4}

Fuente: elaboración propia 
En el módulo 3, equipo de trabajo, se describen los diferentes roles y sus funciones en la construcción de un RED. En este punto, el docente identifica sus funciones como experto temático y comprende la importancia del guion instruccional. Así mismo, el docente identifica las funciones de los demás roles y participación en la construcción de un RED (ver figura 7).

Figura 7. Pantallas del módulo 3, equipo de trabajo
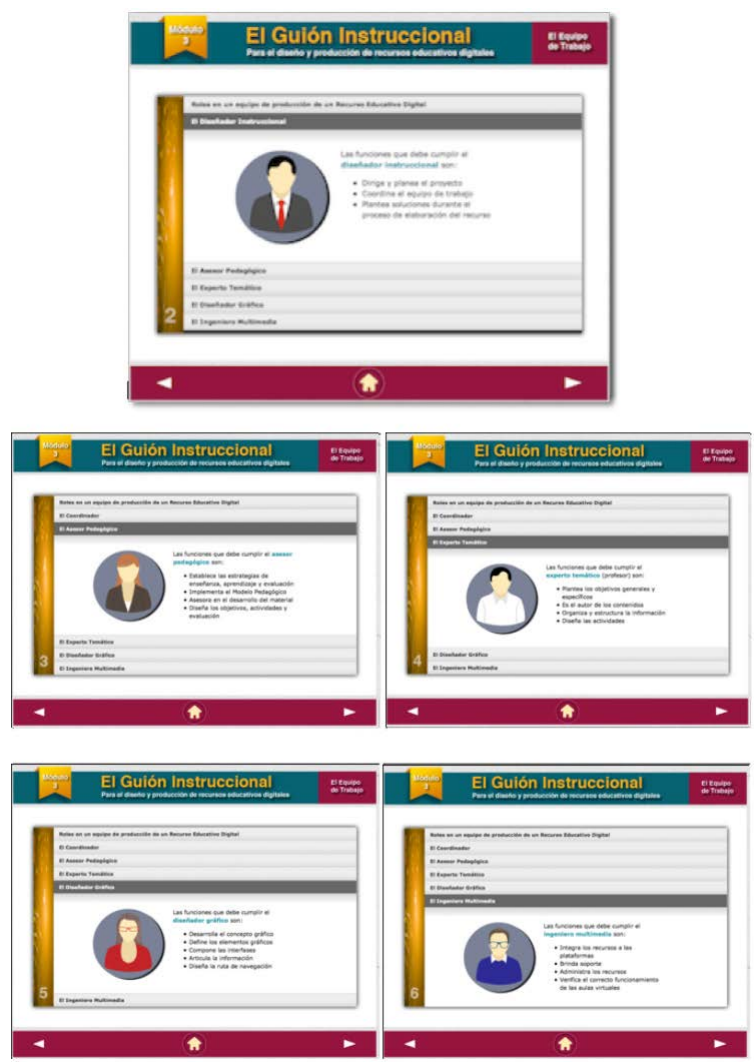

Fuente: elaboración propia 
En la pantalla 2, del módulo 3, se incluye una actividad interactiva en la que el profesor debe identificar y relacionar las funciones de cada rol (ver figura 8). En la parte superior se encuentran los personajes que representan cada rol, en la parte inferior se organizan de manera aleatoria las funciones de todos los roles. El objetivo de esta actividad es identificar cuáles funciones corresponden a cada rol.

Figura 8. Pantalla de actividad interactiva del módulo 3

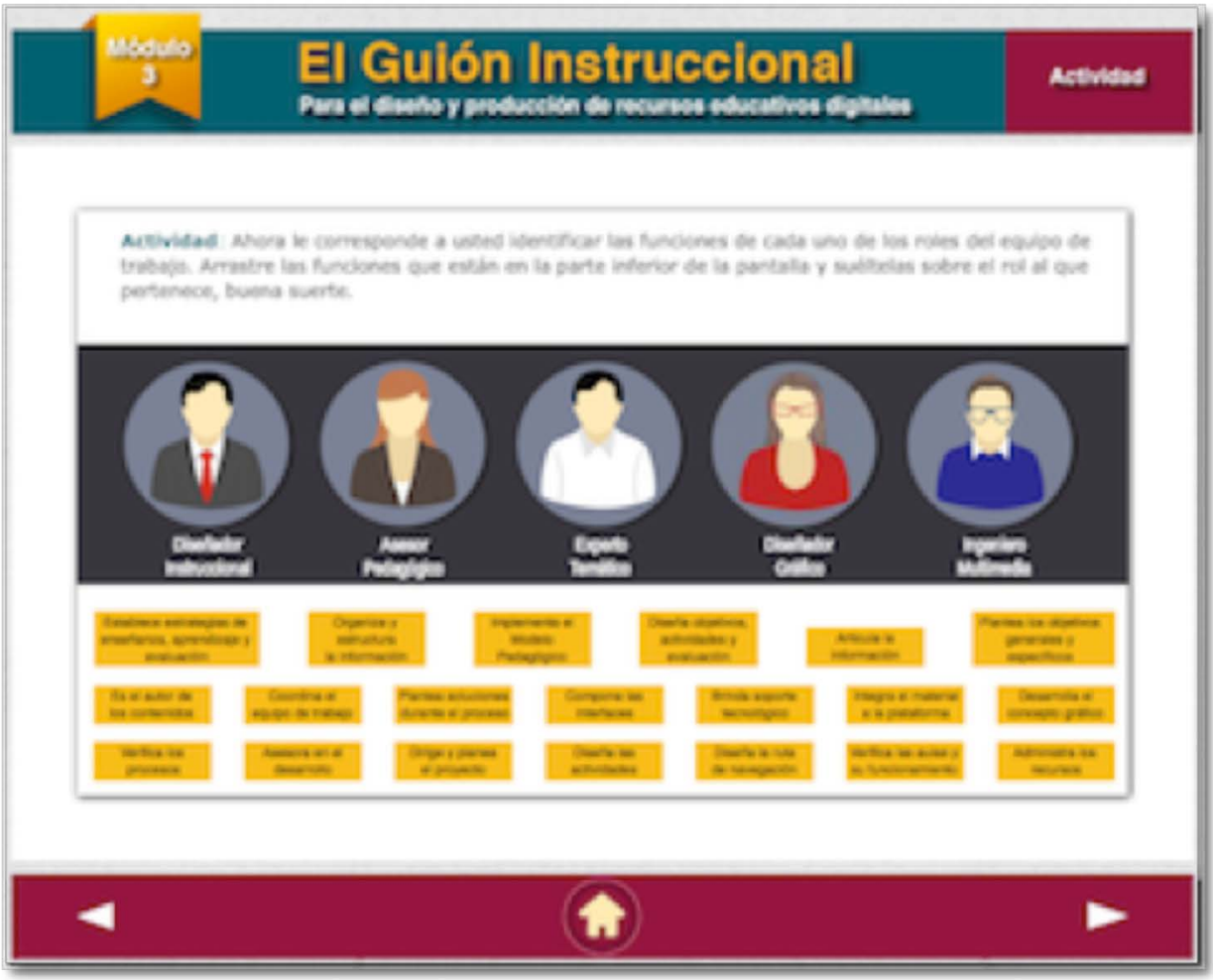

Fuente: elaboración propia 
La actividad consiste en arrastrar con el puntero cada actividad y llevarla hasta el rol que corresponde; un mensaje emergente indicará si es correcto o incorrecto.

El módulo 4, guion instruccional, define y describe el guion instruccional, la función, los factores y pautas para la elaboración y la adecuación pedagógica. En la página 2 del módulo 4 se muestra un formato para la elaboración del guion instruccional y un enlace externo a un repositorio de formatos para la elaboración de un guion instruccional, al ubicarse sobre cada área del formato emerge una imagen con su respectiva descripción (ver figura 9).

Figura 9. Pantallas del contenido del módulo 4
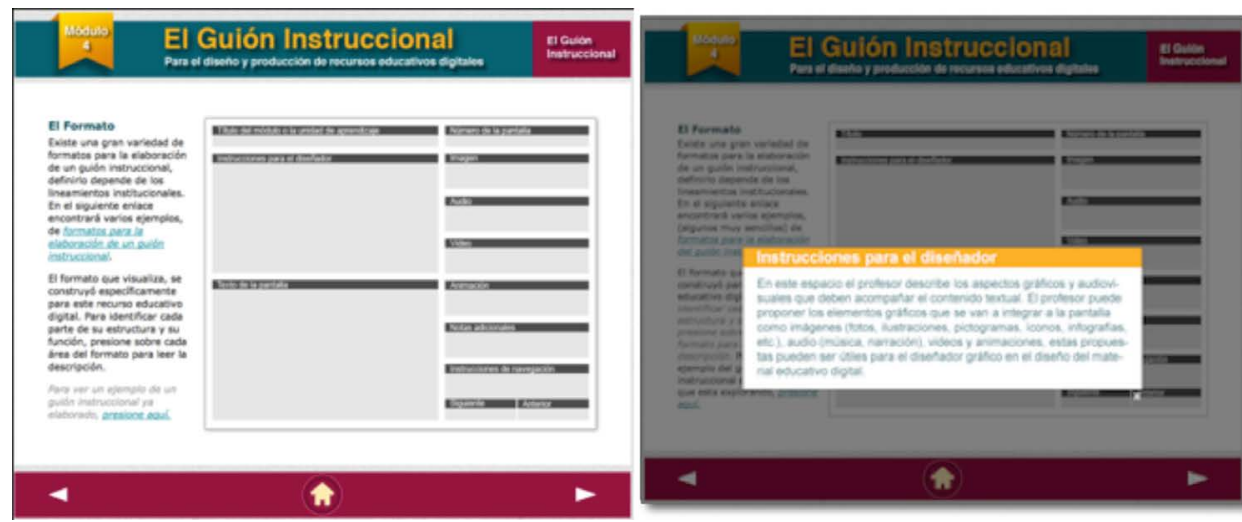

Fuente: elaboración propia

En la página 3 y 4 del módulo 4, se exponen dos ejemplos del guion instruccional de este RED; se inserta una imagen de la pantalla para que el profesor la identifique y la relacione con la información incluida en el formato del guion instruccional; al ubicarse sobre cada una de las áreas del formato la imagen se maximiza para que el profesor lea cómo se elaboró cada ítem.

El módulo 5, manos a la obra, corresponde a la actividad de elaborar el guion instruccional. Al presionar sobre la imagen se puede descargar el formato, diseñado exclusivamente para este recurso (ver figura 10). 
Figura 10. Pantallas del contenido del módulo 5

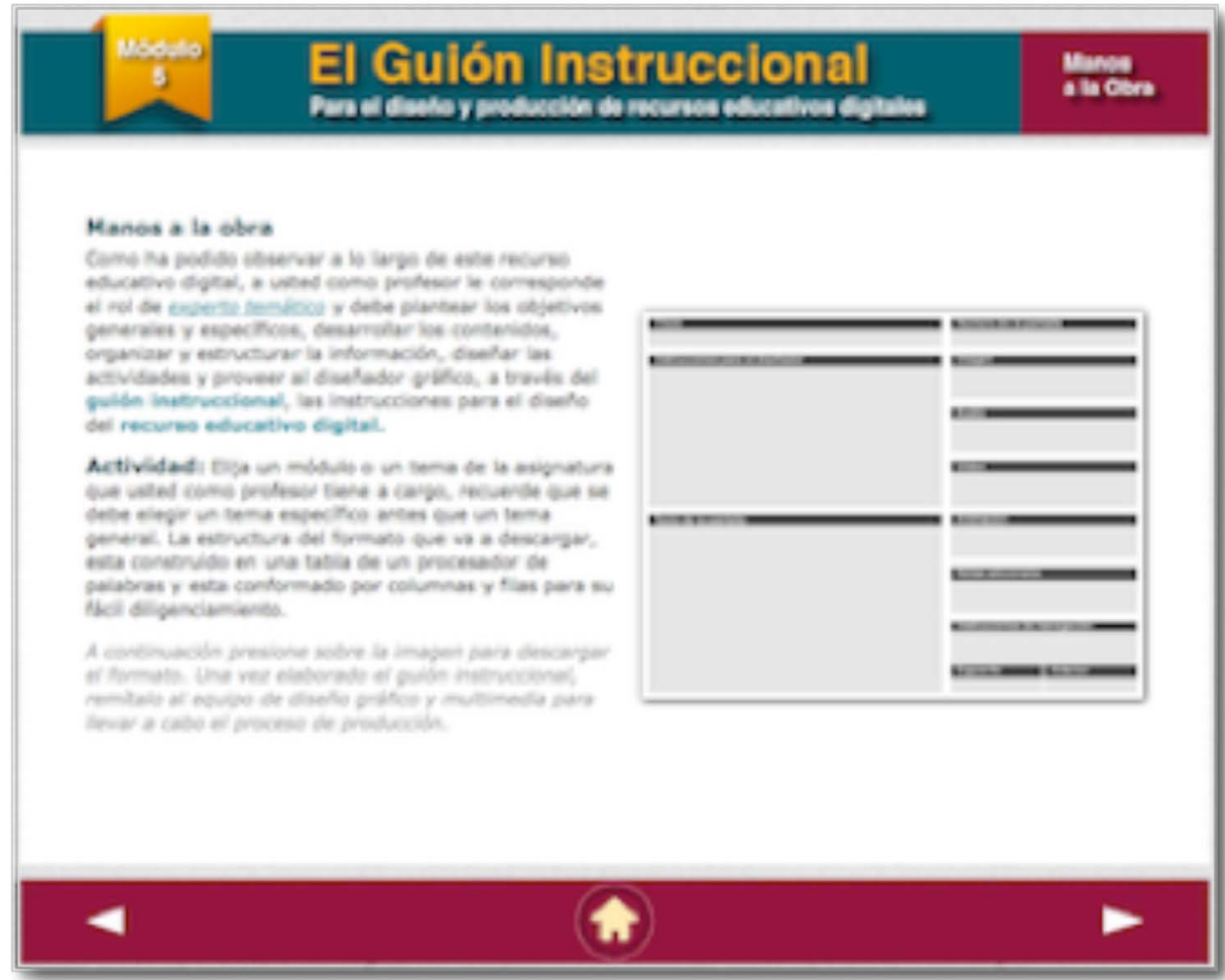

Fuente: elaboración propia

El módulo 6 corresponde a la evaluación que está compuesta por cinco preguntas, cada una con su respectiva retroalimentación. Una vez contestada la evaluación, esta arroja un puntaje y un nivel de aprendizaje. Las preguntas están relacionadas con el concepto de RED y el concepto de guion instruccional. La evaluación permite medir la apropiación de estos dos conceptos, debido a que son indispensables para la correcta elaboración del guion instruccional.

Con respecto a los aspectos tecnológicos, el ambiente virtual de aprendizaje tadeísta AVATA cuenta con la infraestructura necesaria para poner en marcha el uso de 
los RED dentro del aula virtual, plataforma educativa (Moodle), computadores, herramientas para la producción de audio y video y herramientas digitales (con licencia y de uso libre), mesa de ayuda y personal especializado. Todos estos elementos brindan un acompañamiento constante al profesor.

\section{Conclusiones}

Al evaluar el recurso educativo digital (RED) como herramienta guía en la elaboración del guion instruccional en docentes de la Universidad Jorge Tadeo Lozano, se encuentra que es una herramienta eficaz para la capacitación de los docentes en su acercamiento al uso de herramientas tecnológicas y desarrollo de contenidos educativos apoyados por las TIC.

Este se convierte en un recurso que provee información de tipo conceptual, más allá de la acción netamente operativa del docente, lo que lleva a que se convierta no solo en una herramienta de orientación procedimental, sino que, también, sea una ayuda de formación en el quehacer docente. Por lo anterior, se observa que el recurso permitió una reflexión con detenimiento, en cuanto a cómo se debe organizar la información con finalidades pedagógicas y didácticas para lograr mejores recursos educativos como apoyo a la clase presencial.

Para el docente, el RED es una herramienta de capacitación constante muy positiva que permite la retroalimentación en procura del proceso de aprendizaje y autoaprendizaje. Sin embargo, el trabajo realizado muestra que los docentes, en su mayoría, no conocen de antemano lo que es un guion instruccional, por lo que, así mismo, tampoco conocen las bondades del mismo en pro de elaborar un recurso educativo digital. Si los docentes no conocen la existencia y los beneficios de estos recursos, es casi imposible que se tengan elaboradas, de forma masiva, RED que apoyen la docencia y, por ende, el sistema educativo en general.

Este tipo de desconocimiento, lleva a reflexionar acerca de lo necesario que resulta la denominada alfabetización digital. Esta alfabetización es más que necesaria, pues los docentes que no se encuentran actualizados en ella, pierden espacios para lograr 
mejores y mayores objetivos de enseñanza y, por ende, la formación integral en sus aulas de clase, sean físicas o virtuales. Una evidente muestra de esta analfabetismo digital se observó cuando, de los nueve participantes, solo los que presentaron una experticia o conocimiento previo en herramientas tecnológicas y gráficas desarrollaron a cabalidad el guion instruccional para ser calificado de acuerdo con la rúbrica de categorización y calificación de este. Es evidente, entonces, que la experiencia en el uso de herramientas tecnológicas y gráficas facilita la elaboración del guion instruccional.

Este nuevo tipo de analfabetismo ya no se presenta en los ciudadanos de muy bajos recursos que no tienen la oportunidad de acceder a una escuela o institución educativa, sino que se presenta en los profesionales que llevan una vida laboral exitosa en una alta calificación escolar. Se puede ver que los participantes del estudio presentaban, en el nivel mínimo de escolaridad, el nivel de especialización, es decir, que no eran recién egresados o que no tuvieran el momento para desarrollarse laboralmente. Se puede decir que la experticia docente también está planteada pues, incluso, había docentes con más de diez ańos de experiencia en la docencia. La pregunta resultante es cómo hacen sus actividades escolares diarias y cómo trabajan el proceso de enseñanzaaprendizaje. Si el trabajo docente aún se está haciendo del modo tradicional no implica necesariamente que se esté haciendo mal; pero, sin ninguna duda, se está desperdiciando el potencial de las TIC.

Un aspecto fundamental de realizar la alfabetización digital en los profesores es la motivación que se logra en el estudiante, una vez el docente incluye las tecnologías de la información como una herramienta diaria en el proceso de formación integral.

Exponer el RED a un grupo de profesores con experiencia en el uso de herramientas graficadoras y a la vez exponer el RED a un grupo de profesores sin experiencia en el uso de herramientas graficadoras indicó que el RED es un elemento facilitador para quien lo conoce y lo entiende y que, a su vez, puede llegar a ser un elemento denso y dispendioso que desgasta al profesor al momento de diligenciar el guion instruccional. La facilidad entonces está en el aprovechamiento del RED, debido al conocimiento y conceptualización del mismo, más allá de lo operativo que pueda ser. 
Mientras que los guiones instruccionales de los profesores que tienen conocimiento previo fueron calificados en categorías sobresaliente en su mayoría, los profesores que no tenían este conocimiento, prácticamente, no tuvieron calificación o esta fue igual a reprobado, por lo que, de forma redundante, se debe mencionar que tener conocimientos previos en el manejo de herramientas graficadoras, facilita la elaboración del guion instruccional.

La tan esperada planeación docente, de la cual se esperan resultados y logros formativos, no necesariamente está presente en todas las actividades del profesor universitario. En profesionales capacitados esta planeación de sus cátedras se ve obstaculizada por la falta de una visión más amplia de la utilización y conocimiento de recursos educativos.

El recurso permite un avance que redunda en la efectividad y uso de las aulas virtuales, dejando a un lado la calidad de repositorios y buscando que todo este esfuerzo a futuro derive en la conformación de un banco de RED como objetos virtuales de aprendizaje, videos, video tutoriales, entre otros.

Realizar este proyecto permitió identificar la necesidad de que los docentes conozcan las herramientas digitales que se encuentran disponibles en la web para su uso libre y la forma de integrarlas en sus RED a través del guion instruccional.

En el ejercicio de la enseñanza y el aprendizaje, es satisfactorio corroborar que no se necesita ser licenciado en Educación para saber enseñar, que la práctica y la búsqueda de lo innovador y de las herramientas que nos ofrecen las nuevas tecnologías nos permiten ser capaces de transmitir la información, rompiendo con los esquemas tradicionales. Desde esta postura, toma validez el concepto del aprendizaje centrado en el estudiante, en el que las TIC se convierten en ese aliado estratégico que permite desarrollar el aprendizaje significativo y autónomo en el estudiante.

En el ejercicio de la docencia, es necesario trabajar arduamente con los docentes por un cambio o transformación de sus prácticas educativas en el aula. Los docentes deben comprender que, en la actualidad, se requiere de nuevos esquemas y que las TIC ya están inmersas en el ámbito educativo, por lo que deben ser aprovechadas. 


\section{REFERENCIAS}

Bou, G. (1997). El guion multimedia. Madrid: Editorial Anaya.

Cabero, J. (2007). "Las necesidades de las TIC en el ámbito educativo: oportunidades, riesgos y necesidades”. Tecnología y Comunicación Educativas, 45 (21), pp. 5-19.

Campuzano Ruíz, A. (1992). Tecnologias audiovisuales y educación. Una visión desde la práctica. Madrid: Ediciones Akal.

Coll, C. (2004). "Psicología de la educación y prácticas educativas mediadas por las tecnologías de la información y la comunicación. Una mirada constructivista”. Revista Electrónica Sinéctica, 25, pp. 1-24.

Dorrego, E. (2004). Transformación de la educación superior en América Latina. Madrid: Pearson-Prentice Hall.

Drucker, P. (1993). The New Society New Brunswick. New York: Transaction Publishers.

Duart, J. M. \& Sangrá, A. (2000). Formación universitaria por medio de la web: un modelo integrador para el aprendizaje superior. Barcelona: Gedisa y Ediciones de la Universidad Oberta de Catalunya.

Flórez, G. (2014). "La validez jurídica de los documentos electrónicos en Colombia a partir de sus evolución legislativa y jurisprudencial". En Revista Verba Iuris. N.o 31. Enero Junio 2014. (p. 43-71)

Hernández-Sampieri, R., Fernández-Collado, C., \& Baptista-Lucio, P. (2006). Metodología de la Investigación. México, D. F.: McGraw-Hill.

Lara, J. D. (2011). "Sociedad del conocimiento en medio de una sociedad del desconocimiento”. Nómadas, 4 (32), 1-33. 
Moreno, A. \& Velásquez, D. (2015). “Aspectos de la regulación TIC en Colombia”. En Revista IUSTA. N. ${ }^{\circ} 43$, julio-diciembre de 2015, pp. 139-157.

Orihuela, J.L. \& Santos, M.L. (2000). Introducción al diseño digital. Madrid: Anaya Multimedia.

Peter, J. (2004). Nuevas Tecnologías y Educación. Madrid: Pearson.

Puentes, J (2016). Análisis de la apropiación y uso de las TIC por parte de las pymes colombianas en Revista IUSTA. N. ${ }^{\circ} 46$, enero-junio de 2017, pp. 19-44. DOI: http://dx.doi. org/10.15332/s1900-0448.2017.0046.01

Sandoval, L. Y. (2010). "Las TIC en los programas de formación de directivos. Una dimensión humanista para el cambio e innovación tecnológica en las instituciones educativas del nuevo milenio". Conferencia Iberoamericana en Sistemas, Cibernética e Informática.

Sarmiento, L. (2016). Gestión de conocimiento: una apuesta por la calidad institucional en Revista IUSTA. N. ${ }^{\circ} 44$, enero-junio de 2016, pp. 99-109.

Universidad Jorge Tadeo Lozano. (2013). "Plan de capacitación profesoral. Documento de trabajo de la Dirección de Innovación Académica”. Bogotá.

Vygotsky, L. S. (1988). El desarrollo de los procesos psicológicos superiores. Barcelona: Editorial Crítica. 